\title{
Self-Assembly: From Nanoscale to Microscale Colloids
}

\author{
S. C. Glotzer, M. J. Solomon, and N. A. Kotov \\ Dept. of Chemical Engineering, University of Michigan Ann Arbor, MI 48109 \\ DOI 10.1002/aic.10413 \\ Published online in Wiley InterScience (www.interscience.wiley.com).
}

\section{Introduction}

$\mathrm{S}$ elf-assembly is a fundamental mechanism by which structures form in materials. Over the past decades, a primary focus has been to relate materials' behaviors to the spatial arrangement of their fundamental building blocks. These building blocks may comprise atoms, molecules, macromolecules and colloidal particles. It is well known that the same chemical substance can have vastly different properties depending on the way its building blocks are arranged-that is, on its crystal structure. For example, carbon atoms arranged in the diamond structure make for the hardest material on earth, whereas in the graphite structure of a charcoal drawing pen, layers of carbon are abraded without effort. The difference between both materials lies in the way atoms are stacked and the type of bond that forms between them. Likewise, colloidal particles might be arranged in a diamond structure. On that scale, this structure makes a material useful not for its hardness, but for its optical properties such as a photonic band gap. ${ }^{1}$

Today, building blocks of exotic shape and functionality are beginning to be engineered at nanometer and micrometer scales. New synthesis and fabrication techniques are being pioneered to make nanoparticles and colloids whose asymmetric shapes and anisotropic interactions provide a spectrum of crystal polymorphs and assembled structures unprecedented in colloid science. Methods now exist for coding instructions for assembly onto individual building blocks, thereby exploiting biomimetic principles of self-organization observed in proteins, for example. These new building blocks will be the "atoms" and "molecules" of tomorrow's materials, self-assembling into unique structures made possible solely by their design. The urgent question for engineers today is: to what extent may the richness of molecular crystal structures be imparted to suspensions of nanoparticles and colloidal "molecules"?

In this Perspective, we compare building blocks being made today at nanometer and micrometer scales and discuss issues important for generating and predicting their self-assembly into novel and useful structures. In the section titled "Effect of

\footnotetext{
Correspondence concerning this article should be addressed to S. C. Glotzer at sglotzer@umich.edu.
}

(C) 2004 American Institute of Chemical Engineers
Shape and Composition on Assembly", we discuss issues of building block shape and composition, and the role of these factors in the self-organization of particles into ordered assemblies. The section titled "Scale Dependent Effects on Assembly" discusses issues of Brownian motion, interparticle forces, and vitrification and gelation. In the section "Building Block Design Rules from Computer Simulation", we describe several simulation methods appropriate for modeling nanoparticle and colloidal self-assembly, and describe examples of computer models of particles "programmed" for assembly by the use of anisotropic interactions. This section is followed by a brief conclusion and outlook.

\section{Effect of Shape and Composition on Assembly}

\section{Available compositions and shapes at nanometer and micrometer scales}

The last several years have witnessed an explosion in the number of different types of nanometer-sized building blocks. A quick literature survey reveals a wide variety of different materials that comprise today's nanoparticles. Included among these are the chalcogenide forms of $\mathrm{Cd}, \mathrm{Pb}, \mathrm{Hg}, \mathrm{Fe}, \mathrm{Ni}, \mathrm{Co}, \mathrm{Ag}$, $\mathrm{Au}, \mathrm{Cu}$, and $\mathrm{In}$; nitrides and arsenides formed from $\mathrm{Ga}, \mathrm{Si}, \mathrm{Ge}$, Ti; elementary Au, Ag, Pt, Pd, Rh, Si, Ge, Co, Ni, Fe, S, C; and oxides of $\mathrm{Al}, \mathrm{Si}, \mathrm{Ge}, \mathrm{Fe}, \mathrm{Ni}, \mathrm{Co}, \mathrm{Cr}, \mathrm{B}$, Os, Ce, Zn, Zr, Ti. There are also many examples of nanoparticles composed of two or more materials combined together to form core-shell particles (e.g., $\mathrm{SiO}_{2}$-coated $\mathrm{Au}$ particles) or more complex structures. The diversity of particle shapes is similarly extensive, largely due to the crystalline nature of the particles. In addition to rods, wires, and core-shell semiconductor and metal particles, new synthesis techniques demonstrate the possibility of making rings, cubes, tetrapods, triangular prisms, and many other exotic shapes (Figure 1a-1d). Such shapes constitute a virtual zoo of "nano-animals" including aptly named nanoacorns, nano-centipedes, nano-guitars, nanosquids, nanovials and nanowhiskers. ${ }^{2-6}$

In contrast, the comparable list of colloidal particle compositions and shapes is significantly more limited. Self-assembly requires particles with narrow distributions in size and shape. Materials for which monodisperse colloidal particles are easily synthesized is small: typical examples include silica and polymers such as polystyrene (PS) and poly(methyl methacrylate) 

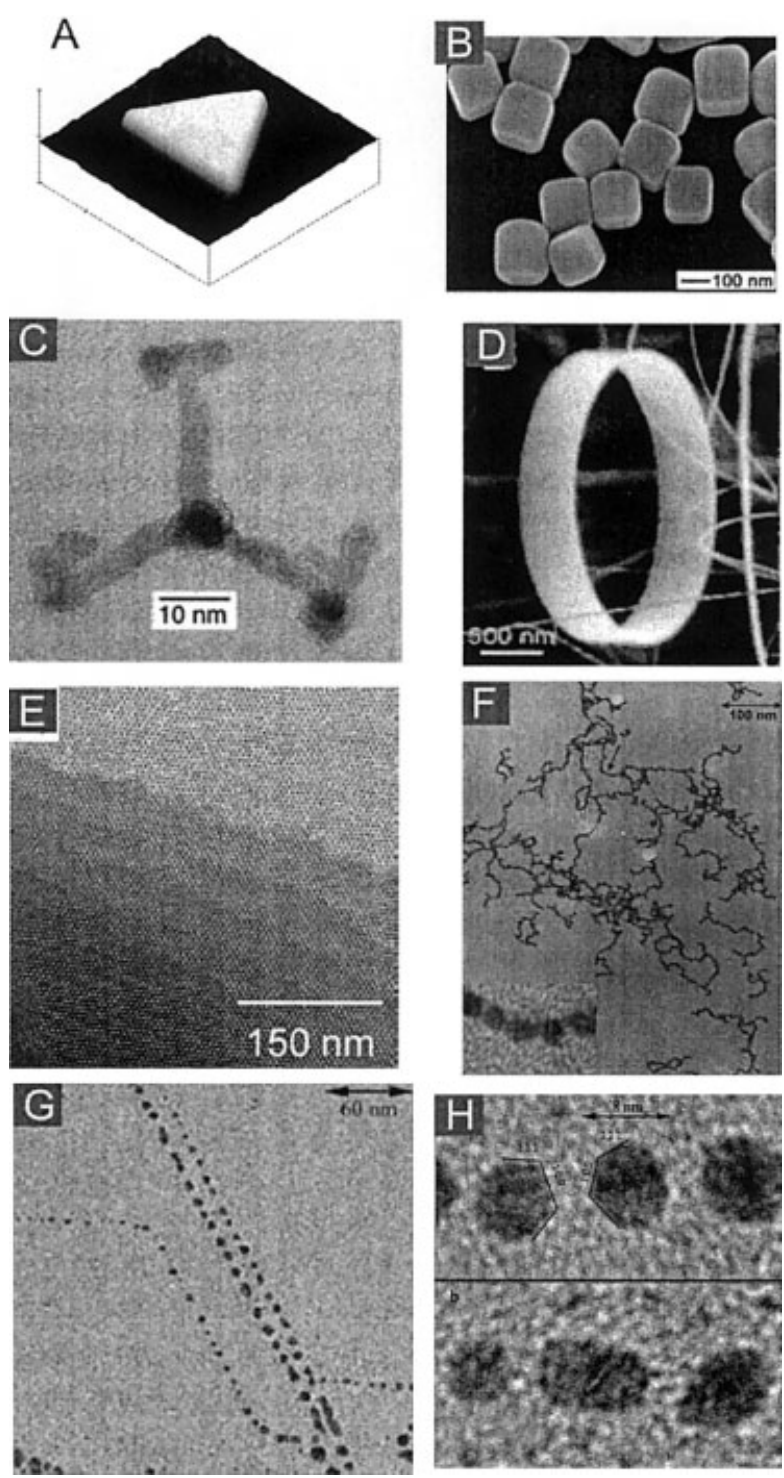

Figure 1. Nanoparticle shapes and nanoparticle assemblies.

(a) AFM image of a triangular Au prism:32 (b) SEM image of silver cubes; ${ }^{35}$ (c) TEM image of a CdSe tetrapod; 50 (d) SEM image of a $\mathrm{ZnO}$ nanoring; 51 (e) TEM of a 3-D array of $3.5 \mathrm{~nm}$ CdSe nanoparticles with a face-center-cubic (fcc) superlattice;52 (f) TEM images of CdTe nanoparticle chains obtained by self-assembly of stabilizer-depleted CdTe nanoparticles; ${ }^{22}$ (g) TEM image of Ag nanoparticle chains, ${ }^{23}$ (h) HRTEM image of the crystallographic orientation in Ag nanoparticle chains..$^{23}$

(PMMA). Such colloids are typically spherical; however, anisometric colloids have been directly synthesized in certain metal oxide systems. ${ }^{7,8}$ Recently, synthesis techniques have been developed to form more complex polyhedral building blocks from spherical particles, ${ }^{9}$ or by stretching spheres into ellipsoids. ${ }^{10-12}$ Other methods to prepare anisotropic particles include microcontact printing, selective deposition and interfacial reaction. ${ }^{13-18}$ Colloids have also been assembled in vesiclelike structures at the surface of emulsions. ${ }^{19}$ These new shapes have the potential to profoundly expand the range of ordered structures possible with colloids.
The great variety of available nanoscale building blocks is beginning to rival the vast molecular "toolkit" chemists have been constructing for some time. Working at the nanoscale provides larger building blocks potentially capable of selfassembling into larger and more complex systems. It provides even the possibility of patterning traditional spherical colloids with nanoparticles and nanostructured molecules to impart anisotropy to interparticle interactions. While semiconducting nanocrystals assembled into arrays can provide circuitry for nanoscale computers, nanopatterned colloids assembled into a carbon-like diamond lattice could be used as a photonic band gap material to control optical properties. This challenge in nanoscale science and engineering is shifting from making new building blocks, to organizing them into one-, two-, and threedimensional (1-D, 2-D, and 3-D) structures. This next step is critical to exploit these new building blocks for novel materials and devices. Taking this step requires answering the following questions: How can we organize nanoparticles and colloids into more complex structures? What kind of structures do we need, and why? What new properties characterize these struc tures? Within the answers to these questions lie the potential for many interesting discoveries and surprising new phenomena. Inventing methods of self-assembly that allow scale-up for manufacturing will enable these discoveries to profoundly impact technologies in medical, electronics, and computing applications.

\section{Assemblies of nanoparticles and the role of shape and composition}

Direct manipulation of nanocolloids into organized structures using tools such as surface probe microscopy is limited and requires the nanoparticles to be supported on a substrate. In contrast, self-organization in solution of nanocolloids into complex 1-D, 2-D, and 3-D structures by physically encoding the eventual desired architecture and its symmetries into the structure and symmetry of the nanocolloid has potentially unlimited promise. One of the simplest examples of such encoding is the preparation of monodisperse nanoparticles that self-organize into hexagonally packed nanocolloidal crystals ${ }^{20}$ (Figure 1e). The next level of complexity can be seen in the spontaneous formation of chains (Figure 1f) from stabilizer depleted CdTe nanoparticles. ${ }^{21,22}$ An interesting effect that can be observed in this system is the diffusion of photons (called "wave-guiding") along the chains, which may allow for directed transport of light, and which is important for optoelectronics and quantum computing. Chains have also been observed for silver ${ }^{23}$ (Figure $1 \mathrm{~g}-\mathrm{h}$ ), iron oxide, ${ }^{24}$ titanium oxide $^{25}$ and other particles. In the case of silver, wave-guiding has also been observed. ${ }^{26}$ The mechanism of self-organization of nanoparticles into chains is understood for CdTe and partially understood for iron oxide nanoparticles. In the former, strong electric dipoles with a magnitude as high as $100 \mathrm{D}$ are formed in nanocrystals of CdTe due to the anisotropy of the crystal lattice and charge trapping. The energy of attraction between two of these nanocrystals is sufficient to overcome thermal energy, resulting in the spontaneous formation of chains. A similar mechanism, as well as attraction between magnetic dipoles, may underlie chaining of Ag nanocrystals.

The ability to self-assemble can also be imparted to nanocolloids by selective surface modification, e.g., with biological 
ligands. This method of structural encoding is exceptionally powerful because of the wide variety of proteins, DNA and RNA that have specific affinity for each other. This encoding can make possible self-assembly of complex electronic or optical circuits from nanowires and nanoparticles. Recent reports on the preparation of various superstructures with the help of biomolecules provide initial steps in this direction. ${ }^{27-31}$

Broadly speaking, the self-organization of nanocolloids and the complexity of the resulting assemblies is determined by the anisotropy of interactions among the nanocolloids. In this respect, two important ways of imparting anisotropy to interparticle interactions are through particle composition and particle shape. With regards to composition, at present there is a very limited list of nanoscale colloids with heterogeneous "microstructure", such as biphasic CoPdS nanoparticles. ${ }^{2,20}$

In contrast, the wide variety of nanoparticle shapes already synthesized allows one to introduce a broad spectrum of anisotropic properties in the nanoscale structures through anisometry. Anisometry can influence optical, magnetic, electrical, biological, and other properties of the nanoparticle itself, and can induce anisotropies in the forces between nanoparticles, and consequently useful asymmetries in the assembled structures. Similar richness may be seen in liquid crystals, where rod-like, disk-like, and ellipsoidal molecules pack into phases with asymmetries that are exploited for displays and other optical devices. The effect of shape anisotropy on the selforganization of particles can be demonstrated by the spontaneous alignment of flat triangular prisms of $\mathrm{Au}$ (Figure 1a) when adsorbed on a polyelectrolyte substrate, ${ }^{32}$ or by the self-organization of $\mathrm{Co}_{3} \mathrm{O}_{4}, \mathrm{Ag}$, and $\mathrm{Au}$ nanocubes ${ }^{33-35}$ (Figure 1a). Computer simulations also demonstrate the effect of shape anistropy on self-assembly of polymer-tethered nanoparticle. ${ }^{36}$

At a practical level, the relationship between particle shape and the ability of particles to self-organize due only to excluded volume and close packing can be understood for simple cases. To predict structures more complex than close packed arrays, the anisotropy of interparticle interactions must by treated more rigorously by mapping the force fields around them. Three points need to be made in this respect. First, various approaches to evaluating interparticle forces may be borrowed from the classical colloidal physics field, modified to treat the wide range of nanoparticle shapes. Second, with suitable force fields and sufficient knowledge about interparticle interactions, computer simulation can be useful for predicting self-assembled structures from collections of particles. Third, the role of size needs to be addressed as the size scales of particles for assembly decrease from micrometers to nanometers. These issues are addressed in the next sections.

\section{Scale Dependent Effects on Assembly}

At the Angstrom scale, molecular assembly has yielded a vast array of functional structures that underpin materials and technologies associated with chemical engineering (e.g., zeolites for catalysis, polymer membranes for separations). Here, common experimental tools for characterization are optical and NMR spectroscopy, neutron scattering and X-ray scattering. Simulation methods involve $a b$ initio quantum mechanics calculations and molecular dynamics. Likewise, large-scale colloidal structures, on the scale of microns, are principal components of materials such as paints, inks and ceramics. Here, the typical characterization tools include light scattering and optical microscopy. Brownian and Stokesian dynamics are the key simulation methods. Nanoscale science and engineering is associated with scales in which the molecular paradigm crosses over to the colloidal paradigm. At the nanoscale, a synthesis of molecular and colloidal approaches will provide new ways to engineer assembly. In pursuit of a synthesis of the two paradigms, we discuss three issues: Brownian motion, interparticle potentials and vitrification and gelation. Each issue highlights the juxtaposition between the molecular and colloidal aspects of assembly. We are particularly interested in assessing ways in which the crossover from molecular to colloidal behavior can be described and exploited for improved assembly at the nanoscale.

\section{Brownian motion}

Colloids undergo random displacement due to their bombardment by impulsive, stochastic forces from the solvent in which they are dispersed. The thermal energy dictates the magnitude of these stochastic forces. The long-time limit of the mean-squared displacement that a free colloidal sphere undergoes due to this Brownian motion is

$$
\lim _{t \rightarrow \infty}\left\langle\Delta r^{2}(t)\right\rangle=6 D_{0} t=\frac{k T t}{\pi \mu a}
$$

Here $D_{O}$ is the Stokes-Einstein diffusivity, $k$ is Boltzmann's constant, $T$ is temperature, $t$ is time, $a$ is the particle radius, and $\mu$ is the solvent viscosity. Long ago it was recognized that this colloidal result is surprisingly robust: it successfully describes diffusion even at nanoscale dimensions. For colloids, a full description requires generalization to a time-dependent diffusivity, inclusion of inertial effects at short times, and incorporation of many-body effects due to thermodynamic and hydrodynamic interactions and their coupling. ${ }^{36}$ Yet, Eq. 1 is sufficient to illustrate the effect of Brownian motion on assembly as the scale is reduced. Consider that at fixed $t, T$ and $\mu, \mathrm{Eq}$. 1 shows that a characteristic displacement normalized on the colloid size scales as $a^{-(3 / 2)}$. For example, all other parameters fixed, a $10 \mathrm{~nm}$ colloid will diffuse 1,000 times further relative to its dimension than a $1.0 \mu \mathrm{m}$ colloid. Alternatively, and perhaps more traditionally, we can examine $t^{*}$, a characteristic time for a colloid to diffuse its own radius. We see that $t^{*} \sim \mathrm{a}^{3}$. These relationships demonstrate that Brownian particles are increasingly delocalized relative to their size upon scaling down from the micro to the nanoscale. The delocalization affects nucleation kinetics, gel and glassy dynamics and the relative strength of fields, such as shear and sedimentation. These phenomena in turn mediate assembly processes. Furthermore, since the particle size often sets the range of potential interactions, the relative scales of Brownian motion and potential interactions are highly dependent on particle size. We consider this point in the next subsection.

\section{Nature, strength and range of potential interactions}

Potential interactions are a key determinant of structural assembly regardless of scale. However, the interaction types of greatest consequence vary significantly as one progresses from molecules to colloids. First, consider molecules. Long-range 


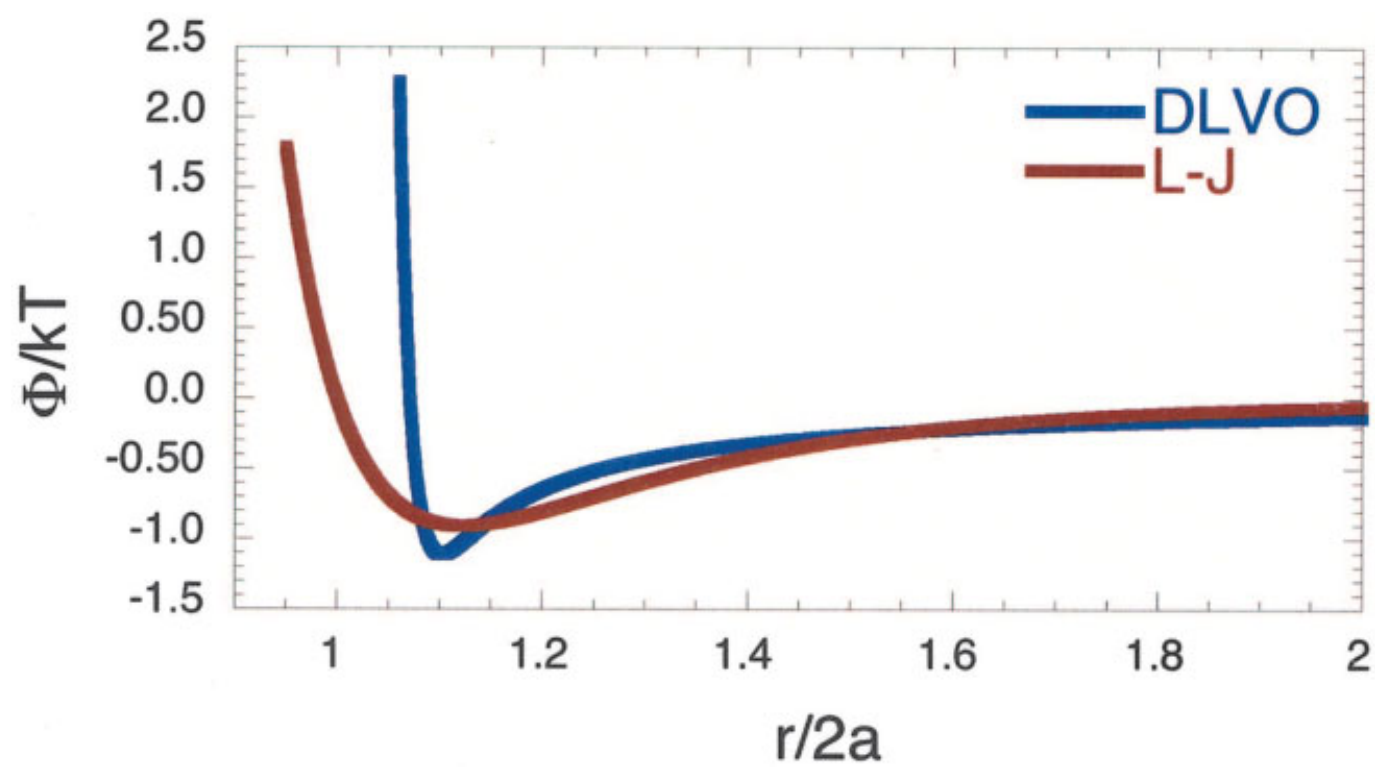

Figure 2. Molecular and colloidal potential interactions.

Under certain conditions, the pair potential interactions between molecules and colloids are of comparable range and magnitude. Here, an example of a molecular interaction is the Lennard-Jones potential plotted at a reduced temperature $\mathrm{T}^{*}=\mathrm{kT} / \varepsilon=1.1$. The colloidal interaction is the DLVO potential between two spherical (dia. $=1 \mu \mathrm{m}$ ) polystyrene colloids dispersed in water. This pair potential shows the secondary minimum for colloids of surface potential $30 \mathrm{mV}$ dispersed in a solvent with Debye layer thickness $\sim 13 \mathrm{~nm}$. The pair potentials are normalized by the thermal energy kT. The separation distances (shown here from particle center to particle center for both potentials) are normalized by the particle diameter, $2 \mathrm{a}\left[{ }^{37,38}\right]$.

weak attraction due to van der Waal's dispersion forces and repulsive excluded volume interactions are approximately captured by, for example, the Lennard-Jones potential. Atoms with isotropic and centrosymmetric potentials of this kind assemble into only a few unit cells of simple symmetry. Of course, multiple species and molecular constituents yield a much richer range of unit cells. Specific, directional interactions (e.g., dipole moments, hydrogen or covalent bonding and ligand binding), and the anisotropic shape of molecules are one origin of this diversity. Now, consider colloids. Here, long-range attractive forces due to the van der Waals interaction exist for particle pairs dispersed in a solvent with dielectric contrast. Excluded-volume interactions due to grafted or adsorbed steric layers are present. If electrolyte is dissolved in the solvent, the repulsive force between two colloids due to the distribution of charge on their surfaces is modeled by a screened Coulombic interaction. These contributions yield, for example, the celebrated DLVO pair potential interaction. ${ }^{36,37}$ Another type of interaction that can be generated between colloidal pairs is the depletion potential: small nonadsorbing polymers dispersed in a solvent induce an attractive potential of mean force between large colloids due to an entropic, free-volume effect. ${ }^{38}$ The depletion interaction is a powerful tool for assembly because its range and strength can be independently manipulated. Other interactions that can be realized include short-range adhesive forces due to changes in steric layer conformation ${ }^{39}$ and induced dipole forces. ${ }^{40}$

Note that with the exception of induced dipole forces, the colloidal pair interactions discussed are isotropic and centrosymmetric, just as the atomistic Lennard-Jones potential is. Figure 2 compares the colloidal DLVO potential (here in the vicinity of its secondary minimum) to the Lennard-Jones interaction. For certain parameter values the correspondence between molecular and colloidal interactions is good when the pair interactions are made dimensionless by the thermal energy, and the range is scaled by a characteristic diameter (atomic or colloidal). In view of Figure 2, it is thus not surprising to learn that self-assembly of bulk 3-D crystals at the colloidal scale is typically limited to structures with simple unit cells, such as face-centered cubic (fcc), body-centered cubic (bcc), and hexagonal close-packed (hcp), just as for monoatomic systems with interactions as in Figure 2. By comparing to molecules, we see that one conceptual way forward to increase the diversity of assembled structures at the nano and colloidal scales is to seek particle building blocks with anisotropic, noncentrosymmetric interactions, as well as anisotropic shape, as discussed in the section titled "Effect of Shape and Composition on Assembly".

Figure 2 and its discussion also illustrate how comparison of the type, strength and range of pair potential interactions on the molecular, nano and colloidal scale demonstrates the way in which some thermodynamic limitations on assembly processes can be ameliorated. However, assembly on the nano and colloidal scale also suffers from kinetic limitations. Bulk 3-D crystals grow slowly, contain large coexisting amorphous or glassy regions, and are prone to defects, such as vacancies, stacking faults and grain boundaries. Classical nucleation theory illustrates to some degree the effect of scale on these kinetic limitations. By viewing bulk assembly as crystallization, it is clear that 3-D assembled structures can be induced either heterogeneously (perhaps by means of a surface template) or through the spontaneous formation of small crystal nuclei. Classical nucleation theory describes the rate per unit volume, $I$, of the latter, homogeneous process

$$
I=\kappa \exp \left(-\Delta G_{\text {crit }} / k T\right)
$$


Here, $\Delta \mathrm{G}_{\text {crit }}$ is the height of the free-energy barrier to nucleation, and $\kappa$ is a kinetic prefactor that is best understood for particles with hard sphere interactions. If $I$ is reduced to a dimensionless form by the factor $(2 a)^{5} / D_{0}$, then $\kappa=A \phi^{5 /}$ ${ }_{3} D(\phi)$ where $\mathrm{A}$ is a dimensionless constant, $\phi$ is the amorphous liquid volume fraction, and $\mathrm{D}(\phi)$ is the reduced shorttime self-diffusivity, a generalization of the free particle Stokes-Einstein diffusivity in Eq. 1.41,42 Here, a dimensionless scale for time in the nucleation rate is $a^{2} / D_{0} \sim a^{3}$, exactly the scaling we developed in the Brownian motion section. The cubic exponent indicates the profound kinetic retardation that accompanies the scale up of molecular or small-particle assembly to the dimensions characteristic of the wavelength of light (necessary for optical sensing and photonics applications, for example). Moving beyond hard sphere assembly kinetics, we note that, for systems with pair potentials, such as Figure 2, the strong size effect on Brownian motion will likely have additional implications for assembly kinetics beyond what we have discussed here for hard spheres. These implications should be better assessed.

\section{Glass transition and gelation}

Both molecular and colloidal systems can display a glass transition. Particularly for colloids, this glass transition may suppress crystallization kinetics to the point where thermodynamically feasible pathways to assembly are effectively blocked. Because dynamical retardation that accompanies the glass transition is due to the caging effect of the repulsive excluded volume of surrounding particles, this impediment to assembly is most problematic at high densities close to packing limits. Gelation, however, is more problematic for the engineering of assembly. Gelation, a slowing down of dynamics to which strongly interacting nanocolloids and microparticles are particularly susceptible, is due to strong short-range attractive interactions that trap the system in a nonordered state that may be only metastable. Efforts to design potential interactions with an attractive component of range and magnitude conducive to assembly may, instead, induce nonequilibrium aggregation and gelation. Physical (as opposed to covalent) gelation of this kind has few analogues at the molecular scale-it is truly a problem that has come of age as the frontier of materials development has been extended to the nanoscale. Unusual effects of particle shape on aggregation and gelation will also complicate assembly efforts with building blocks of this kind. ${ }^{43}$ Better understanding of the dynamical or thermodynamic origin of gelation, as well as ranges and strengths of potential interactions for which it occurs, are needed to design strategies to circumvent this problem. Recently, mode coupling theory ${ }^{44}$ and clustering transition thermodynamics ${ }^{45}$ have been used to achieve helpful first steps in this direction.

\section{Building Block Design Rules from Computer Simulation}

\section{Simulation methods for self-assembly of nanoparticles and colloids}

A range of simulation methods exists for investigating aspects of self-assembly of colloidal and nanoparticle suspensions. Monte Carlo (MC) methods are often used for mapping out equilibrium phase diagrams of colloids modeled by hard sphere, soft-sphere, DLVO and other interparticle pair potentials. A stochastic method, MC generates particle configurations probabilistically and may be used to obtain ordered structures in a variety of thermodynamic ensembles. These methods are also useful at molecular and nanometer scales provided appropriate pair potentials are known. The same pair potentials used in MC may be used in molecular dynamics (MD) and Brownian dynamics (BD) simulations. MD generates particle configurations deterministically by solving Newton's second law, $\mathbf{F}_{i}=\mathrm{m}_{i} \mathbf{a}_{i}$, where the force $\mathbf{F}_{i}$ on particle $i$ is obtained from the gradient of the pair potential $\mathrm{U}, \mathrm{m}_{i}$ is the mass of particle $i$ and $\mathbf{a}_{i}$ is the acceleration of particle $i$. In its simplest form, MD generates a microcanonical (constant energy) ensemble of particle trajectories. By adding a thermostat and/or barostat, configurations consistent with the canonical and isothermalisobaric ensembles may be generated. These are often more suitable for comparison with experiments. In an MD simulation of a nanoparticle or colloidal suspension, the solvent must be modeled explicitly, although coarse-grained models may often be used to simplify the computation.

Even with such simplifications, a simulation of, e.g., 1,000 nanocolloids in water self-assembling from an initially disordered state into an ordered array is prohibitive, and, thus, simulators often employ methods in which the effects of solvent are included implicitly rather than explicitly. BD is one such method. ${ }^{46}$ In BD, an effective pair potential (such as a potential of mean force) is used to obtain the conservative force $\mathrm{F}_{\mathrm{i}}^{\mathrm{C}}$ on each particle $i$, due to the other particles in the system. This potential represents the "bare" potential between particles as mediated by the solvent. A drag force $\mathrm{F}_{i}{ }^{\mathrm{D}}$ and random force $\mathbf{F}_{i}^{R}$ acts on each particle to represent the dissipative friction forces and random thermal forces, respectively, imparted to each particle by the solvent molecules. The following equation of motion is solved for each particle

$$
m_{i} \mathbf{a}_{i}=\mathbf{F}_{i}^{C}+\mathbf{F}_{i}^{F}+\mathbf{F}_{i}^{R}
$$

Excluded volume, van der Waals, and Coulomb interactions, screening effects, etc., are explicitly included in $\mathbf{F}_{i}^{C}$. Details of these particle interactions arising from, e.g., particle composition, surface modification, etc. enter through this term. In BD, drag is included through the frictional force, which for individual particles in the absence of hydrodynamic interactions is $\mathbf{F}_{i}^{F}=-\gamma_{i} \mathbf{v}_{i}=-6 \pi a \eta \mathbf{v}_{i}$ where $\gamma_{\mathrm{i}}=6 \pi \mathrm{a} \eta$ the friction coefficient, $\alpha$ is the particle diameter, $\eta$ is the solvent viscosity, and $\mathbf{v}_{i}$ is the particle velocity. The Brownian motion of the particle resulting from the ramdom bombardment of solvent molecules is included through $\mathbf{F}_{i}^{R}$, and can be calculated using the fluctuation-dissipation theorem

$$
\left\langle\mathbf{F}_{i}^{R}(t) \mathbf{F}_{i}^{R}\left(t^{\prime}\right)\right\rangle=6 k_{B} T \gamma_{i} \delta\left(t-t^{\prime}\right)
$$

and the requirement that $\mathbf{F}_{i}^{R}$ have zero mean. We see that the drag force and the Brownian force depend on particle size, which has important implications for assembly as the particle size decreases from micrometer to nanometer scales.

Another mesoscopic method used to model colloidal dispersions is dissipative particle dynamics (DPD). ${ }^{48}$ This method uses soft particles to represent fluid elements, but hard particles must be used to capture the packing effects of colloidal parti- 
cles due to excluded volume, which is important in determining the local order. Although the soft force used to calculate the interactions between the fluid elements expedites the computation by allowing larger times steps in integrating the equations of motion, the overall simulation time likely will be dominated by the excluded volume terms required for local particle packing.

An important challenge in using simulation to predict assembled nanoparticle and colloid structures with any of the above simulation methods is obtaining realistic models to describe the effective interactions in these complex systems. "Minimal" models that incorporate in a crude way attractive and repulsive interactions between particles arising from solvent-phobicity and solvent-philicity, respectively, are useful in providing insight into the types of assembled structures possible. Amphiphilic interactions between anisotropically patterned (or "patchy") particles, ${ }^{49}$ for example, may be modeled using the same level of interaction potentials used successively to map block copolymer, surfactant, and liquid crystal phase diagrams. Refining and parameterizing these potentials requires input from more detailed computations using atomistic classical force fields and either $\mathrm{MD}$ or $\mathrm{MC}$, and $a b$ initio quantum mechanics (QM) calculations. Density functional theory (DFT) QM methods are necessary for obtaining, e.g., the surface structure (e.g., faceting) of a single CdTe quantum dot or Au nanocrystal and for obtaining forces between the organic molecules in the stabilizer coating and the atoms on the surface of the nanocrystal. Atomistic MD may then be used with suitably parameterized force fields to obtain potentials of mean force between nanoparticles in solvent for a system containing several particles.

\section{Simulation studies of patchy particles}

BD simulations of novel nanoparticle and colloidal building blocks using minimal models describing the amphiphilic nature of the particles predict that they should self-assemble under the right conditions into structures atypical of traditional materials. ${ }^{47,48}$ For example, model spherical particles with sticky patches placed on two opposite poles assemble continuously on cooling into chains in a manner similar to that observed in equilibrium polymerization of monomers. ${ }^{48}$ Similar particle chaining has been observed in suspensions of nanoparticle quantum dots. ${ }^{21,22}$ Particles with four sticky patches placed equidistantly on each particle's equator assemble via a discontinuous transition into sheets with square packing; a larger number of patches on the equator can induce different 2-D packings. Model particles with more complex patterns, such as complementary double-ring-like patches shifted off the equatorial plane, were found to self-assemble into tetrahedra, icosahedra, and higher order polyhedra (Figure 3a,b). This approach may provide a strategy for making large numbers of tiny, self-assembling, 3-D nanoscopic electronic circuits ${ }^{49}$ for devices with repeating units, such as memory arrays. Rings of particles are predicted when complementary patches are placed anisotropically on the equatorial plane of each particle at a relative angle of less than $180^{\circ}$ (Figure $3 \mathrm{c}, \mathrm{d}$ ). The diameter of the rings can be controlled by tuning the angle between the patches, suggesting one strategy for making conducting rings for nanoelectronic devices and materials with negative indices of refraction. The recognitive feature of the complementary
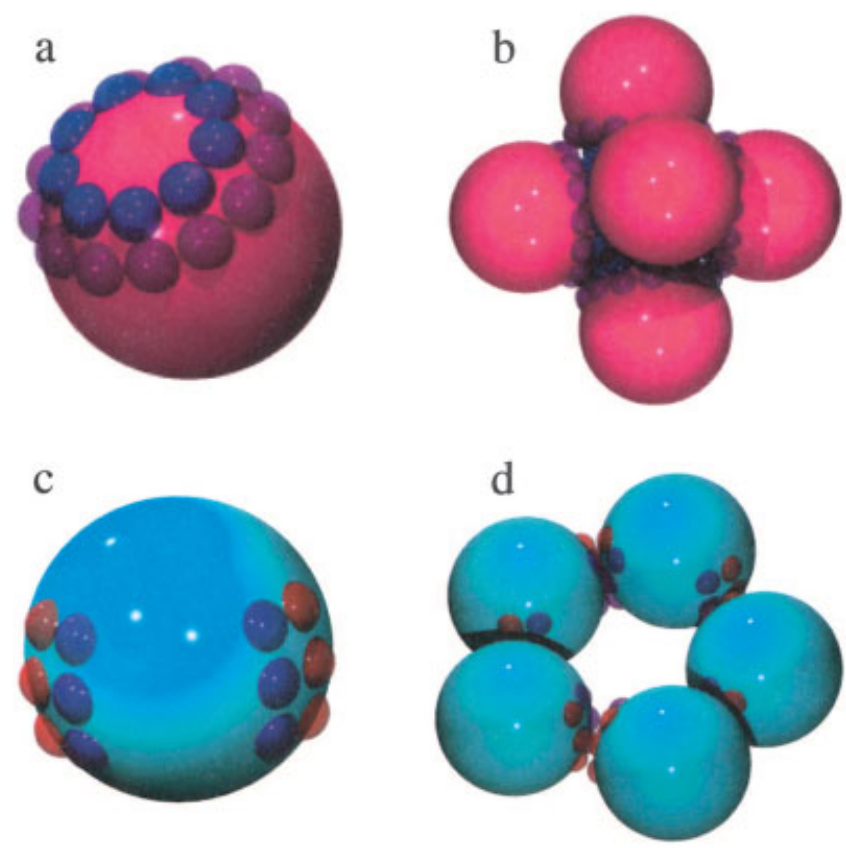

Figure 3. Anisotropically interacting particles and their assemblies predicted by computer simulation.

(a) Spherical particles with two rings of sticky "patches" that interact attractively with like patches on other particles. Here, blue patches interact via a LJ potential, red patches interact via a LJ potential, and red patches interact with blue patches via a soft-sphere excluded volume repulsive interaction. (b) Square pyramid structure assembled on cooling from six particles as in (a). Structure shown is taken from a larger simulation that includes many such structures formed from hundreds of particles. By changing the angle of the patches below the equatorial plane, other polyhedra, such as tetrahedra and icosahedra are obtained. (c) Spherical particle with sticky "patches" that interact attractively with like patches on other particles. The interactions are the same as in (a). (d) Ring assembled on cooling from five particles as in (c) Structure shown is taken from a larger simulation that includes many such structures formed from hundreds of particles. By changing the angle between the patches, rings of larger and smaller diameter may be obtained via self-assembly. See, for example, Ref. 49. Images rendered by C.R. Iacovella.

interactions, such as that found in complementary sequences of DNA, was reported to be necessary in maintaining the correct relative orientation of the particles during assembly. By exploiting particle shape anisotrophy, further complexity in assemblies can be achieved. ${ }^{49}$

\section{Conclusion and Outlook}

This Perspective has identified the enormous potential to exploit shape and interaction anisotropy in nano- and microscale building blocks for assembly. The relationship between building block symmetry and assembled phase morphology has of course long been appreciated at the molecular scale. However, as the building block size is increased from molecular to nano to colloidal scales, we have argued that the diversity of unit cells successfully assembled to date decreases dramatically. Yet, many new reports of the preparation of anisotropic and anisometric building blocks for particle assembly are now appearing, especially at the nanoscale. The challenge now is to develop heuristics and principles to assemble these new build- 
ing blocks into useful 1-D, 2-D and 3-D structures. Toward this end, improved understanding of the effect that building block size has on the ways that Brownian motion and interaction potential mediate self-assembly in solution will allow promising methods developed for a given application to be successful scaled up or down in size for additional technological application. Simulation methods can facilitate such progress by identifying general features of assembly energetics, dynamics and kinetics that are valid regardless of scale, and those that may be unique to a particular range of length and timescales. In this way, distinctions between the theory and practice of assembly in the realms of molecules, nanoparticles and colloids that are apparent today can be replaced instead by one set of universal guiding principles. This fundamental achievement will certainly enhance technological progress in this rapidly evolving area in which chemical engineering is sure to play a central, leading role.

\section{Acknowledgments}

We gratefully acknowledge financial support from the National Science Foundation, Department of Energy, National Aeronautics and Space Administration, National Institutes of Health, Air Force Office of Scientific Research, and the Oklahoma Center for the Advancement of Science and Technology. SCG and MJS thank Mark Horsch, Chris Iacovella, Ali Mohraz, Tesfu Solomon and Zhenli Zhang for discussions and images. NAK thanks Z.Tang, A Syniagin, J. Lee, A Mamedov, Y. Wang, N. Mamedova, L. Liz-Marzen and M. Correa-Duarte.

\section{Literature Cited}

1. Maldovan M, Thomas EL. Diamond-structured photonic crystals. Nature Materials. 2004;3:593-600.

2. Teranishi T, Inoue Y, Nakaya M, Oumi Y, Sano T. Nanoacorns: Anisotropically phase-segregated $\mathrm{CoPd}$ sulfide nanoparticles. JACS. 2004;126(32):9914-9915.

3. Wang Y, Tang ZY, Liang XR, Liz-Marzen LM,. Kotov NA. SiO2-coated CdTe nanowires: Bristled nano centipedes. Nano Lett. 2004;4(2):225-231.

4. Staldar A, Duerig U. Nanoguitar: oscillating string as a force sensor. Review of Scientific Instruments. 1995;66(6): 3576-3579.

5. Litborn E, Roeraade J. Liquid lid for biochemical reactions in chip-based nanovials. J of Chromatography B: Biomedical Sci and Appl. 2000;745(1):137-147.

6. Li B, Y Xie, Huang JX,. Qian YT. Synthesis by a solvothermal route and characterization of CuInSe2 nanowhiskers and nanoparticles. Adv Materials. 1999; 11(17):14561459.

7. Buining PA., Pathmamanoharan C, Jansen JBH, Lekkerkerker HNW. Preparation of colloidal boehmite needles by hydrothermal treatment of aluminum alkoxide precursors. JACS. 1991;74:1303.

8. Thies-Weesie D, Philipse A, Kluijtmans S. Preparation of sterically stabilized silica-hematite ellipsoids: Sedimentation, permeation, and packing properties of prolate colloids. J. Colloid Interface Sci. 1995;174:211-223.

9. Manoharan VN, Elsesser MT,. Pine DJ. Dense packing and symmetry in small clusters of microspheres. Science. 2003;301:483-487.

10. Keville KM, Franses EI, Caruthers JM. Preparation and characterization of monodisperse polymer microspheroids. J. Colloid Interface Sci. 1991;144:103-126.

11. Ho CC,. Keller A, Odell JA, Ottewill RH. Preparation of monodisperse ellipsoidal polystyrene particles. Colloid. Polym. Sci. 1993;271:469-479.

12. Jiang P, Bertone JF, Colvin VL. A Lost-wax approach to monodisperse colloids and their crystals. Science. 2001; 291:451-457.

13. Fujimoto K, Nakahama K, Shidara M, Kawaguchi H. Preparation of unsymmetrical microspheres at the interfaces. Langmuir. 1999;15:4630-4635.

14. Takei H, Shimizu N, Gradient sensitive microscopic probes prepared by gold evaporation and chemisorption on latex spheres. Langmuir. 1997;13(7):1865-1868.

15. Nakahama K, Kawaguchi H, Fujimoto K. A novel preparation of nonsymmetrical microspheres using the langmuir-blodgett technique. Langmuir. 2000;16:7882-7886.

16. Lu Y, Xiong H, Xia Y, Prentiss M, Whitsides GM. Asymmetric dimers can be formed by dewetting half-shells of gold deposited on the surfaces of spherical oxide colloids. J. Am. Chem. Soc. 2003;125:12724-12725.

17. Love JC,. Gaters BD,. Wolfe DB, Paul KE, Whitsides GM. Fabrication and wetting properties of metallic half-shells with submicron diameters. Nano Lett, 2002;2:891-894.

18. Cayre O, Paunov VN, Velev OD. Fabrication of asymmetrically coated colloid particle by microcontact printing techniques. J. Materials Chem. 2003;13:2445-2450.

19. Dinsmore AD, Hus MF,. Nikolaides MG, Marquez M, Bausch AR, Weitz DA, Colloidosomes: selectively permeable capsules composed of colloidal particles. Science. 2002;298:1006-1009.

20. Talapin DV, Koeppe R,. Goetzinger S, Kornowski A, Lupton JM,. Rogach AL,. Benson O, Feldmann J, Weller H. Highly emissive colloidal $\mathrm{CdSe} / \mathrm{CdS}$ heterostructures of mixed dimensionality. Nano Lett. 2003;3(12):1677-1681.

21. Tang Z, Wang Y,. Ozturk B, Kotov NA, One-dimensional energy transfer in CdTe nanoparticle chains. J. Phys. Chem. 2004;108(22):6927-6931.

22. Tang ZY, Kotov NA, Giersig M. Spontaneous organization of single CdTe nanoparticles into luminescent nanowires. Science. 2002; 297(5579):237-240.

23. Giersig M,. Pastoriza-Santos I, Liz-Marzan LM. J. Materials Chem. 2004;14: 607-610.

24. Iakovenko SA, Trifonov AS, Giersig M,. Mamedov A, Nagesha DK,. Hanin VV,. Soldatov EC, Kotov NA. Oneand two-dimensional arrays of magnetic nanoparticles by the Langmuir-Blodgett technique. Adv Materials. 1999; 11(5):388-392.

25. Polleux J, Pinna M, Antonietti M, Niederberger M, $A d v$ Materials. 2004;16:436-439.

26. Maier SA, Kik PG,. Atwater HA. Phys. Rev. B. 2003;67: 205402-1 - 205402-5.

27. Wang SP, Mamedova N, Kotov NA, Chen W, Studer J. Antigen/antibody immunocomplex from CdTe nanoparticle bioconjugates. Nano Lett. 2002;2(8):817-822.

28. Dujardin E, Peet C, Stubbs G, Culver JN, Mann S. Nano Lett. 2003;3:413-417. 
29. Moghaddam MJ, Taylor S, Gao M, Huang S, Dai L, McCall MJ. Nano Lett. 2004; 4:89-93.

30. Mokari T, Rothenberg E, Popov I, Costi R, Banin U. Science. 2004;304:1787-1790.

31. Sun Y, Mayers B, Herricks T, Xia Y, Nano Lett. 2003;3: 955-960.

32. Malikova N, Pastoriza-Santos I, Schierhorn M,. Kotov A, Liz-Marzan LM. Layer-by-layer assembled mixed spherical and planar gold nanoparticles: control of interparticle interactions. Langmuir. 2002;18(9):3694-3697.

33. Xu R, Zeng HC. Self-generation of tiered surfactant superstructures for one-pot synthesis of $\mathrm{Cu} 3 \mathrm{O} 4$ nanocubes and their close- and non-closepacked organizations. Langmuir. 2004;20(22):9780-9790.

34. Yu D, Yam VW-W. Controlled synthesis of monodisperse silver nanocubes in water. JACS. 2004;126(41):13200-13201.

35. Sun Y, Xia Y. Shape-controlled synthesis of gold and silver nanoparticles. Science. 2002;298:2176-2179.

36. Zhang ZL, Horsch MA,. Lamm MH, Glotzer SC. Tethered nano building blocks: toward a conceptual framework for nanoparticle self-assembly. Nano Lett. 2003;3(10): 13411346.

37. Russel WB, Saville DA, Schowalter WR. Colloidal Dispersions. Cambridge: Cambridge University Press; 1989.

38. Israelachvili J. Intermolecular and Surface Forces. 2nd ed. London: Academic Press; 1992.

39. Poon W. The physics of a model colloid-polymer mixture. J Physics - Cond Mat. 2002;33:R859-R880.

40. Grant MC, Russel WB. Volume-fraction dependence of elastic moduli and transition temperatures for colloidal silica gels. Phys Rev E. 1993;47(4):2606-2614.

41. Yethiraj A, van Blaaderen A. A colloidal model system with an interaction tunable from hard sphere to soft and dipolar. Nature. 2003;513-517.
42. Auer S, Frenkel D. Prediction of absolute crystal-nucleation rate in hard-sphere colloids. Nature. 2001;409:10201023.

43. Harland JL, van Megen W. Crystallization kinetics of suspensions of hard colloidal spheres. Phys Rev E. 1997; 55:3054-3067.

44. Mohraz A, Moler D,. Ziff RF, Solomon MJ. Effect of monomer geometry on the fractal structure of colloidal rod aggregates. Phys. Rev. Lett. 2004;92(155503):1-4.

45. Dawson KA. The glass paradigm for colloidal glasses, gels and other arrested states driven by attractive interactions. Curr Op Coll Int Sci. 2002;7:218-227.

46. Dudowicz J, Freed K, D JF. Flory-Huggins model of equilibrium polymerization and phase separation in the stockmayer fluid. Phys Rev Lett. 2004;92(045502):1-4.

47. Allen MP, Tildesley DJ. Computer Simulation of Liquids. Oxford: Clarendon; 1987

48. Frenkel, D, Smit B. Understanding Molecular Simulation. London: Academic Press; 2001.

49. Zhang ZL, Glotzer SC. Self-assembly of patchy particles. Nano Lett. 2004;4(8): 1407-1413.

50. Gracias DH, Tien J, Breen TL,. Hus C, Whitesides GM. Forming electrical networks in three dimensions by self assembly. Science. 2000;289:1170-1172.

51. Manna L, Scher EC, Alivisatos AP. Synthesis of soluble and processable rod-, arrow-, teardrop-, and tetrapodshaped CdSe nanocrystals. JACS. 2000;122(51):1270012706.

52. Kong XY, Ding YZ, Yang RT, Wang ZL. Single-crystal nanorings formed by epitaxial self-coiling of polar nanobelts. Science. 2004;303:1348-1352.

53. Talapin DV, Shevchenko EV, Kornowski A, Gaponik N, Haase M, Rogach AL, Weller H. Adv Materials. 2001;13: $1868-1871$. 